\title{
The effect of de-gassing on the dispersion of fine oil droplets in water
}

\author{
M.J. Francis, R.M. Pashley* \\ Department of Chemistry, The Australian National University, Canberra ACT 0200, Australia \\ Received 21 July 2005; received in revised form 1 March 2006; accepted 7 March 2006 \\ Available online 22 March 2006
}

\begin{abstract}
Several recent studies have demonstrated that a wide range of hydrophobic, water-insoluble oils can be readily dispersed in water following a simple freeze-thaw process of de-gassing. The oil droplets typically appear to be sub-micron sized and are fairly mono-disperse. They do not coalesce but slowly phase-separate or settle under gravity. This behaviour applies to a wide range of oils including, hydrocarbons, fluorocarbons and silicones. There is also some evidence that finely divided hydrophobic solid powders can also be more readily dispersed in de-gassed water. The enhanced dispersion is thought to be caused by the removal of gas nucleation solutes that is dissolved gas molecules, which would otherwise facilitate cavitation between two hydrophobic surfaces. The almost complete removal of dissolved gases appears to prevent this cavity formation and so hydrophobic particles are more easily dispersed in water. This new area has some interesting applications to several important industries. Some recent results are presented and a simple theoretical explanation is proposed to explain the fine distribution of droplet sizes observed.

(C) 2006 Elsevier B.V. All rights reserved.
\end{abstract}

Keywords: Dispersion; Emulsion; De-gassing

\section{Introduction}

Direct application of the DLVO theory of colloid stability suggests that fine, micron-sized oil droplets should be stable once dispersed in water [1]. The question then is how can oil droplets be dispersed or conversely, what prevents the easy dispersal of oil droplets in water. This issue has been studied in several recent papers, which have demonstrated that de-gassing mixtures of oil and water facilitates oil droplet dispersion in the aqueous phase [1-4]. In a typical experiment, a mixture of about $1 \%$ oil was almost completely de-gassed by a sequential process of freeze-thaw and pumping. In each case the oil was water-insoluble and hydrophobic. It was generally observed that following several freeze-thaw, pumping cycles, the aqueous phase became visually turbid, due to dispersion of oil droplets. In some cases these 'spontaneous' dispersions were studied and in others the mixtures were vigorously shaken for a few seconds or allowed to phase separate first and then shaken. The turbidity and droplet sizes were then studied for various oils and compared with the 'blank' gassed case, that is, where the mixtures were purged with nitrogen gas, to maintain a neutral $\mathrm{pH}$, and then

\footnotetext{
* Corresponding author.

E-mail address: richard.pashley@anu.edu.au (R.M. Pashley).
}

vigorously shaken. The de-gassed dispersions were in all cases, substantially more turbid than for the blanks. Similar enhanced dispersion was also observed for finely powdered hydrophobic solids, dispersed in de-gassed water $[1,4]$.

In each case the turbidity decreased with time, over periods of many hours to days, depending on the density of the oil. Light scattering studies indicate that the oil droplets do not coalesce, as expected from DLVO theory, and that the reduction in turbidity is caused by the droplets phase-separating under gravity [2]. In some experiments turbidity curves, with time, were found to be the same for samples immediately shaken after degassing or when shaken after settling, in sealed vacuum tubes $[1,4]$. That the same dispersion is produced by settled samples, when vacuum-sealed, indicates that the absence of dissolved gas is critical. Further, when a hydrocarbon oil (squalane) and water were separately de-gassed and then mixed and shaken, similar turbidity results were observed to the mixtures de-gassed together [2]. These experiments are also repeated here. Recently, enhanced dispersion has also been reported by the use of degassed water on a hydrophobic powdered material [4] and a lesser enhancement for de-gassed water with gassed oil [3]. These combined results support the explanation that de-gassing causes this enhanced dispersion.

A more detailed explanation for the effect of de-gassing was proposed in a recent report [3]. The argument was based 
on the fact that the separation of two hydrophobic surfaces in water should, thermodynamically [5], cause the intervening film to cavitate, a process observed experimentally [6,7]. However, this cavitation seems possible in normal, gassed water (containing $1 \mathrm{mM}$ of dissolved oxygen and nitrogen) but is inhibited by de-gassing, which presumably removes nucleation sites and increases the activation energy for cavitation. Removal of this cavitation apparently facilitates the dispersion of hydrophobic colloids in water. Once dispersed, oil particles become charged by the selective adsorption of hydroxyl ions [8] and remain stable to coalescence, even when dissolved gases are re-introduced $[1,2]$.

The importance of de-gassing becomes clear when it is realized that the enhancement of dispersion of oil in water is not a limited observation but appears to be a property of the entire class of hydrophobic, water-insoluble oils and hydrophobic powders. So far, studies have been reported on hydrocarbons, fluorocarbons and silicones [1-4]. Other oils should also behave in a similar manner, given the criteria developed later in this paper. Because of this generality, as well as the important potential applications of this enhanced dispersion, such as in drug delivery [4] and surfactant-free cleaning [3], we examine here the possible mechanisms involved.

A recent paper [9] has cast doubt on the role of de-gassing on this enhanced dispersion, apparently because of the observation (originally reported in the first paper, ref. [1]) that dispersion occurs 'spontaneously' during the cyclical freeze/thaw process. This work claims that the process of repeated freezing, in liquid nitrogen, and thawing can cause enhanced dispersion, even without de-gassing, or at least pumping. However, this is not a simple proposal, because it is well-known that freezing causes a degree of de-gassing and is often used in chemical laboratories as a basic method to de-gas liquids. That the freezing part of the process used in the original study aids de-gassing is also supported by the fact that the diffusion of oxygen and nitrogen in water is very slow [2] and de-gassing would be very inefficient without freezing. Freezing liquids exclude solutes from the solid lattice and this will create local de-gassing. To add further complication, the incidental mixing processes associated with bubble formation/de-gassing and the large temperature differentials involved in the freeze-thaw process, will also, of course, enhance any oil droplet dispersion. Burnett et al. [9] claim that this gas exclusion, upon freezing, can be reversed by purging the thawed mixture with nitrogen gas, after each cycle. However, this reversal is not at all clear because gas bubbling can obviously only be introduced into the mixture after it has melted and it has been established that exposure to dissolved gases after the dispersion has formed has no effect [2]. The formation of a cloudy dispersion becomes visible as soon as the mixture begins to melt and so we cannot be sure that bubbling forces molecular dissolved gases into the freshly melted liquid before dispersion can occur. Dissolved gases diffuse only very slowly through water [2]. It should also be noted that copious bubbling occurs naturally during the first few freeze-thaw, pump cycles and that the presence of these bubbles obviously did not prevent the enhanced dispersion. Lastly, it was shown earlier [2] and in the present work, that enhanced dispersion occurs when the liquids are separately de-gassed, showing that only de-gassing is necessary.

Many studies have now demonstrated the importance of degassing, by itself, and it therefore seems reasonable to conclude that the freeze-thaw method combined with pumping produces a de-gassed mixture, partly due to the freezing process and partly due to the pumping. The freeze/thaw process does produce dispersion but the mechanism appears to be related to local de-gassing caused by freezing and the bubbling/thermal mixing involved in thawing. Further evidence that de-gassing is the cause of enhanced dispersion is also given in the present work.

The issue of purity also needs to be considered. A recent paper [9] criticized the lack of further purification of the various oils used 'as purchased' in the original studies. However, it should be noted that further purification had no effect on the results [9]. It should be pointed out that additional purification was considered un-necessary in the original study because any contaminant surfactant in the oils would only have served to raise the turbidity of both samples, reducing any difference between the two.

\section{Materials and methods}

Perfluorooctyl bromide, perfluorohexane and squalane were the purest grades available and were used as supplied, only exposing the liquids to air inside a laminar flow cabinet. Water was prepared by activated charcoal and reverse osmosis filtration prior to distillation and storage in Pyrex vessels in a laminar flow filtered air cabinet.

Mixtures of various oils (typically about 1\%) and water, and the separated liquids, were all de-gassed by a process of repeated freezing in liquid nitrogen in a tube sealed by a Teflon tap, followed by pumping down to a pressure of 0.01 mbar (with the tap open) and then melting, with the tap closed, after the tube was pumped down to the target pressure. Any dissolved gas produced on each melting cycle was removed upon re-freezing and pumping. Although this process was carried out five times, typically no further bubbling, on melting, was observed after three to four cycles. The vacuum pressure of $0.01 \mathrm{mbar}$ corresponds to a de-gassing level of about $99.999 \%$, if it is assumed that the final pressure achieved after several cycles of freeze/thaw/pumping is given by the pressure in equilibrium with the final frozen liquid, which on being melted does not give any visible bubbling or out-gassing.

Dispersion of oil in water was usually achieved by vigorous shaking of the mixture for $8 \mathrm{~s}$ in a sealed Pyrex tube. However, in some experiments where mixtures of oil and water were degassed together, no additional shaking was carried out and the socalled 'spontaneous' dispersion was studied and compared with vigorously shaken samples. In other experiments the mixtures were de-gassed together and then vigorously shaken. Also, some mixtures were separately de-gassed, mixed under vacuum and then shaken. Turbidity was measured using an HF Scientific Micro 100 Turbidimeter. Particle sizes and zeta potentials were measured using a Malvern Zetasizer.

The enhancement of oil droplet dispersion in water is most easily monitored using turbidity measurements. This enhancement can be measured by the difference between the new system 
(de-gassed) and the gassed blank, following vigorous shaking. In each case, the gassed blank was a mixture of the same composition as the de-gassed sample, but was purged with nitrogen gas via a clean Pasteur pipette for $10 \mathrm{~min}$. Turbidity is a measure of how many droplets are dispersed in a given phase and is measured in NTU (nephelometric turbidity units). In the results presented here the NTU value was measured via light scattering. To give an understanding of the magnitude of these turbidity values, distilled water has a turbidity of $0.02 \mathrm{NTU}$, while tap water has a value of 1-5 NTU. Although useful, NTU measurements are of limited value and the results can be inaccurate if the refractive index of the dispersed phase is close to that of the dispersing phase (such as with perfluorocarbons in water). For example, visually turbid samples can give low NTU values because their opacity is caused by light absorption rather than scattering. Hence, in some cases dynamic light scattering (DLS) has been used to obtain the droplet size distribution, as well as the charge on the oil droplets. Careful interpretation of the DLS results is also required for poly-disperse samples. Monodisperse samples show size distribution by volume graphs (see later) over similar size ranges to the Z-average (diameter) and have a small PDI value (poly-dispersity index). The magnitude of the PDI is a measure of poly-dispersity and for poly-disperse samples the $\mathrm{Z}$-average is accepted as the best estimate of average droplet size.

Interfacial tension values for various oils in water were determined using the drop profile method.

\section{Results}

\subsection{The dispersion properties of perfluorohexane}

The effect of de-gassing on the dispersion of perfluorohexane $(\mathrm{PFH})$ is illustrated in the photograph in Fig. 1, where

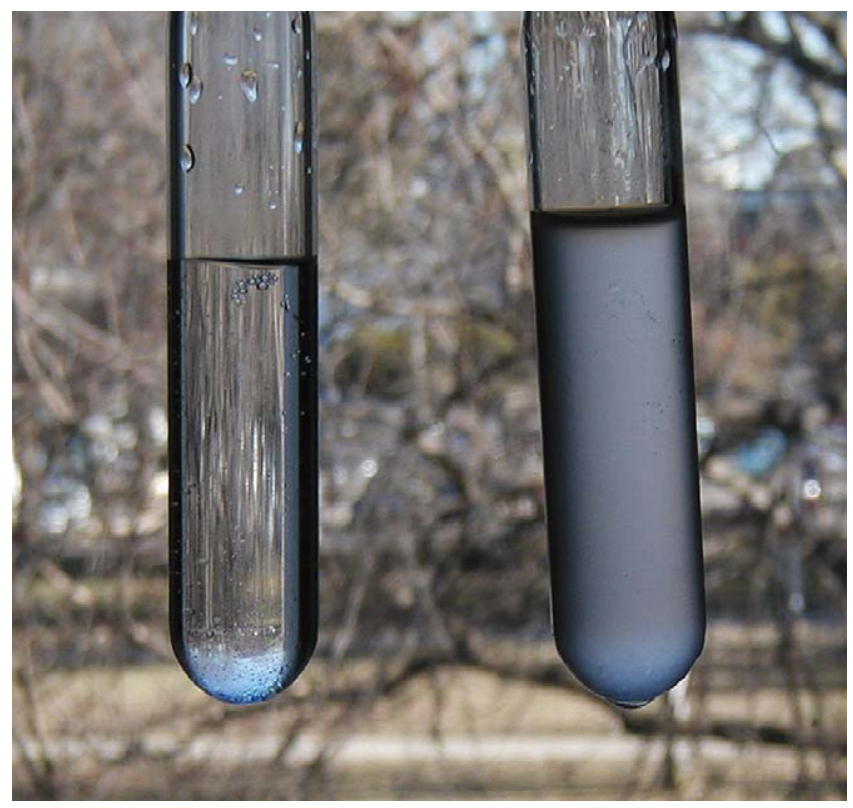

Fig. 1. $0.2 \mathrm{ml}$ of PFH dispersed in $25 \mathrm{ml}$ of water (de-gassed on right, gassed on left); taken 2-3 min after $10 \mathrm{~s}$ of vigorous shaking. the turbidity of samples vigorously shaken for $8 \mathrm{~s}$, immediately after de-gassing, and the corresponding blank samples, nitrogen purged (to give a $\mathrm{pH}$ of 7) were compared a few minutes after vigorous shaking. The gassed mixture had two separate phases, whereas the de-gassed mixture was readily dispersed and maintained its stability for many hours. The difference in turbidity is striking because of the similarity in refractive index of the oil (1.29) and water (1.33). It is clear that the gassed, blank samples did not produce fine droplets, whereas the de-gassed mixtures immediately produced a fine, uniform distribution of particles, which remained stable for may hours. The droplets produced in the de-gassed mixture settled under gravity and this settling rate was monitored. The settling times observed corresponded, using Stokes law, to the average droplet sizes measured by DLS for similar fluorocarbon oil, PFOB (see later).

The shaken, de-gassed mixture was left in a glass heat-sealed tube under vacuum for many days. The de-gassed dispersion was mostly clear after about $15 \mathrm{~h}$. After several days the phases completely separated to give a clear aqueous and a clear, lower fluorocarbon phase. However, some fine white droplets were visible on the surface of the lower fluorocarbon phase. This fine 'mist' was also witnessed in the dodecane dispersion when it was de-gassed and sealed in the same manner. This mist was shaken slightly to re-disperse it in the bulk phase without disturbing the bulk of the oil that had by this stage phase separated. The turbidity, however, was much lower than for when most of the oil was dispersed in the aqueous phase, yet given the small size of the droplets that do not re-coalesce with the oil phase this is not unexpected. Vigorous shaking, after several days, produced a very similar turbidity to the original de-gassed sample.

In further experiments on perfluorohexane ( $\mathrm{PFH})$ and water, the pure $\mathrm{PFH}$ and water phases were separately de-gassed. The de-gassed water was then added to the de-gassed PFH oil and the mixture was shaken, under vacuum. The oil was found to disperse in the aqueous phase, as shown in Fig. 2. In this case there was no frozen or thawed interface between the oil and water and yet significantly enhanced dispersion was still observed.

A series of experiments were also carried out using the two tube apparatus with several different conditions of de-gassing and mixing of PFH and water. The turbidity results obtained after vigorous shaking are summarized in Fig. 3. De-gassing the PFH only and mixing with gassed (nitrogen purged) water yields a more stable and turbid dispersion than when the water is degassed and the PFH is purged with nitrogen, yet both are above the control (where both phases were gassed). This is surprising, as the ratio of water to $\mathrm{PFH}$ is $125: 1$ and $\mathrm{PFH}$ can only hold about $20 \times$ the amount of dissolved gasses compared to water. De-gassing of the fluorocarbon oil appeared to be important. The highest turbidity was obtained when both oil and water were (separately) de-gassed and mixed under vacuum, although the case where PFH and water were de-gassed in separate vessels and then mixed under a nitrogen atmosphere, was also found to be similar.

In another series of experiments the effects of varying the number of freeze-thaw/pumping cycles was measured. After each series of cycles the PFH/water mixture was vigorously shaken and its turbidity monitored over time. The results in Fig. 4 


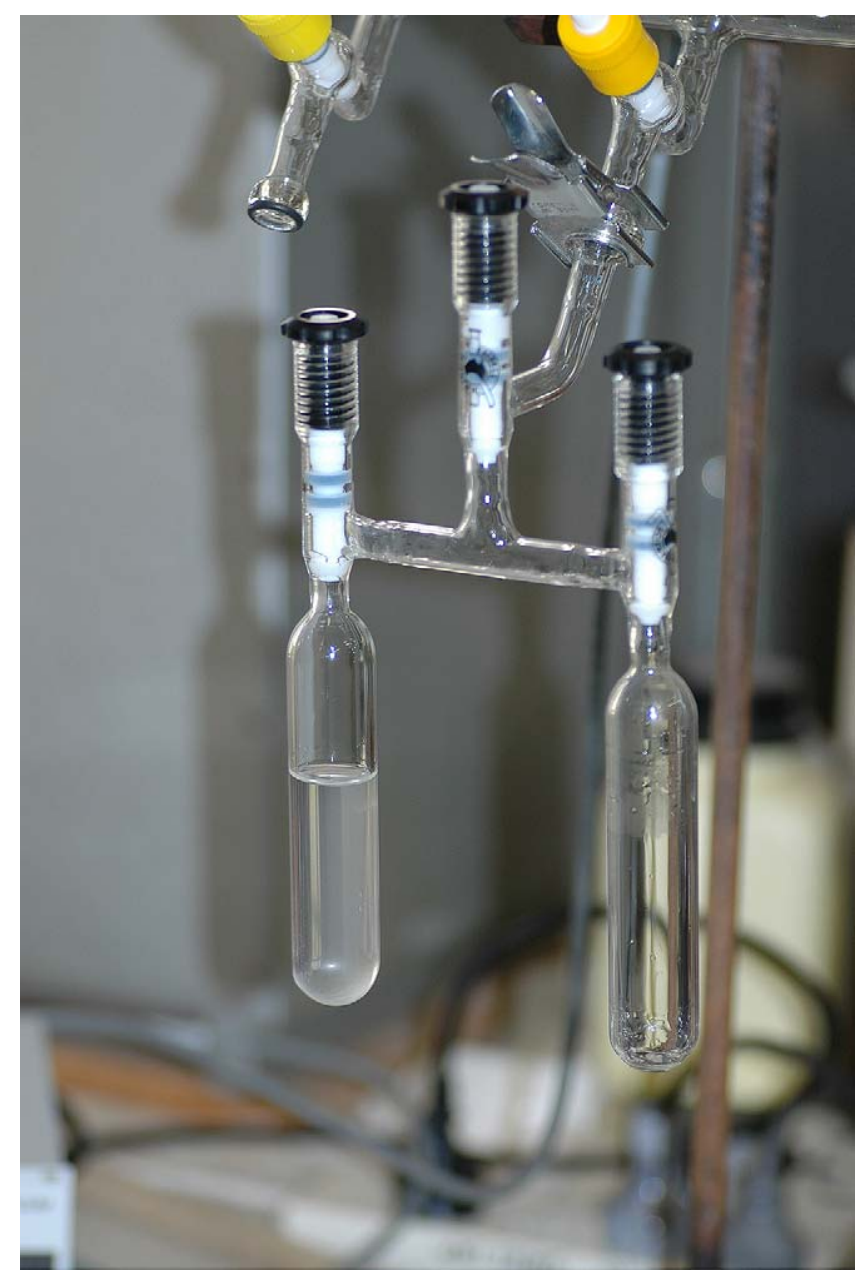

Fig. 2. Three tap apparatus. Separately de-gassed water was added to de-gassed PFH and the photo was taken 2-3 min after shaking. The central tap was kept closed to keep the system sealed under vacuum.

demonstrate that each freezing cycle increases the turbidity of the mixture, until the point of complete de-gassing, where bubbling no longer occurs on thawing. At this point, typically after four cycles, the turbidity curves remain the same, with no further increase.

$0.2 \mathrm{ml}$ of PFH and $25 \mathrm{ml}$ prepared seperately then mixed

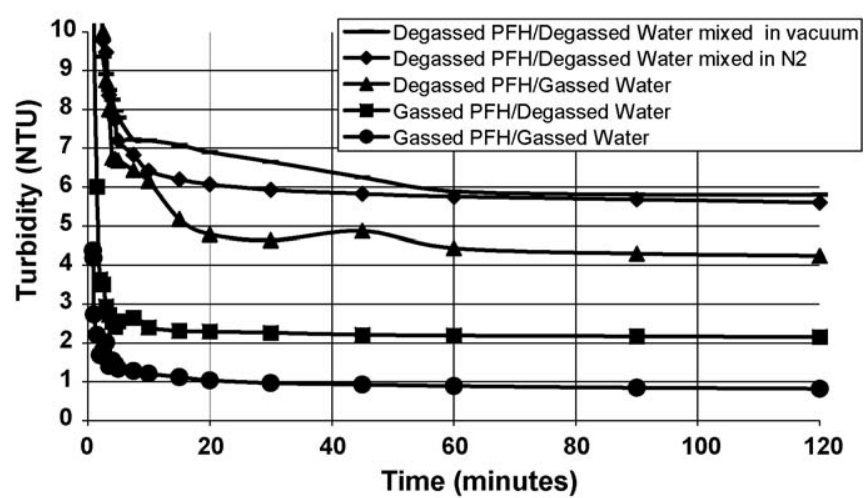

Fig. 3. $0.2 \mathrm{ml}$ of PFH and $25 \mathrm{ml}$ of water prepared separately (either purged or de-gassed) then mixed under vacuum or in a nitrogen-purged atmosphere. In all cases 'gassed' refers to nitrogen purging for $10 \mathrm{~min}$ to exclude dissolved $\mathrm{CO}_{2}$.
$0.2 \mathrm{mI}$ PFH and $25 \mathrm{mI}$ Water degassed together with different number of cycles

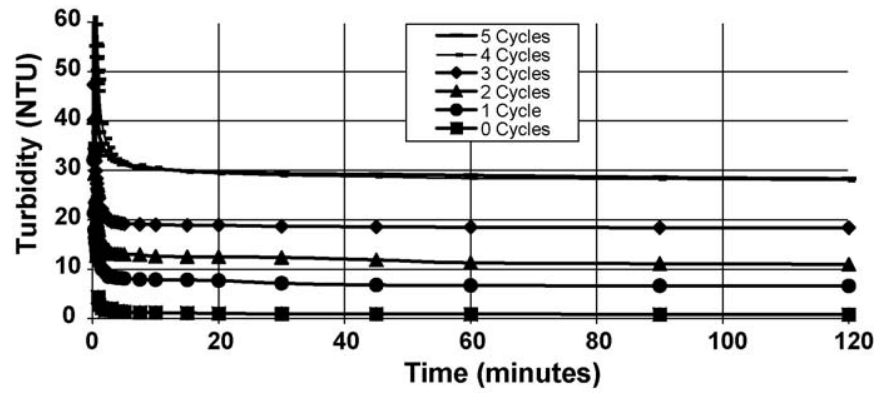

Fig. 4. $0.2 \mathrm{ml}$ of PFH dispersed in $25 \mathrm{ml}$ of water then de-gassed via various freeze-thaw cycles.

The effect of increasing the $\mathrm{pH}$ of the aqueous phase was studied because of the observation that oil droplets are stabilized by the specific adsorption of hydroxyl ions [8]. Initial studies showed that increasing the $\mathrm{pH}$ from 7-10 caused a three times increase in turbidity of the dispersion. This increase in stability was achieved by introducing a hydroxyl salt; however, with a similar concentration of fluoride salt (being isoelectric with hydroxyl salts) no greater stability was witnessed. These results support the proposed explanation, that hydroxyl ion adsorption stabilizes the dispersion.

\subsection{The dispersion properties of squalane}

The high molecular weight hydrocarbon oil squalane, which was used in earlier studies was also examined as a comparison with the fluorocarbon liquids. The two tube apparatus was used to study the effect of de-gassing carried out separately and as a mixture. The results are shown in Fig. 5, where turbidities in each case were measured over time following vigorous shaking. For this hydrocarbon oil, the turbidities observed for separate de-gassing of the liquids were similar to those obtained for the de-gassing of the mixture. This is in agreement with results published earlier [2]. The 'spontaneous' dispersion, with no shaking, appears to produce a similar dispersion of fine droplets, that is with similar turbidity values to the shaken samples, after significant settling times, of say $1 \mathrm{~h}$. The initial turbidities were

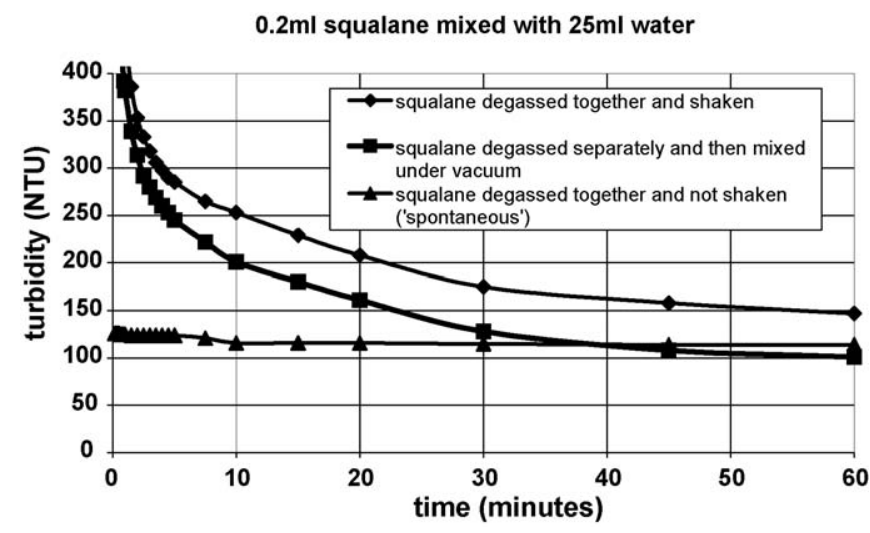

Fig. 5. $0.2 \mathrm{ml}$ squalane dispersed in $25 \mathrm{ml}$ of water under different de-gassing scenarios. 
Table 1

Physical properties of some common hydrocarbon and fluorocarbon oils

\begin{tabular}{|c|c|c|c|c|}
\hline & Castor oil & \multicolumn{2}{|c|}{ Soybean oil } & Hexadecane \\
\hline \multicolumn{5}{|c|}{ Physical properties of some natural oils } \\
\hline Interfacial tension $(\mathrm{mN} / \mathrm{m})$ & 3.5 & \multicolumn{2}{|l|}{15.0} & 53.8 \\
\hline Surface tension $(\mathrm{mN} / \mathrm{m})$ & 39.0 & \multicolumn{2}{|l|}{25.0} & 27.5 \\
\hline Density $(\mathrm{g} / \mathrm{ml})$ & 0.96 & \multicolumn{2}{|c|}{0.916} & 0.77 \\
\hline \multirow[t]{2}{*}{ Theoretical contact angle $\left({ }^{\circ}\right)$} & 60.5 & \multicolumn{2}{|c|}{82.0} & 111 \\
\hline & $\mathrm{C}_{7} \mathrm{~F}_{16}$ & $\mathrm{C}_{6} \mathrm{~F}_{14}$ & $\mathrm{C}_{8} \mathrm{~F}_{18}$ & Perflubron \\
\hline \multicolumn{5}{|c|}{ Physical properties of several fluorocarbons } \\
\hline Interfacial tension $(\mathrm{mN} / \mathrm{m})$ & 39.7 & 38 & 42 & 47 \\
\hline Surface tension $(\mathrm{mN} / \mathrm{m})$ & 12.85 & 11.91 & 14.00 & 18 \\
\hline Density $(\mathrm{g} / \mathrm{ml})$ & 1.75 & 1.669 & 1.73 & 1.93 \\
\hline Theoretical contact angle $\left({ }^{\circ}\right)$ & 112 & 111 & 113 & 113 \\
\hline
\end{tabular}

much higher for the shaken samples than for the 'spontaneous' samples, as reported earlier [1].

The interfacial tension of squalane and water was measured using the droplet profile method. The value obtained was $37.0 \pm 0.3 \mathrm{~mJ} \mathrm{~m}^{-2}$. This value is similar to that reported for perfluorohexane (see Table 1).

\section{Discussion}

\subsection{The role of de-gassing in dispersion}

The results presented here on the dispersion of mixtures of separately de-gassed liquids, squalane and water and PFH and water, clearly demonstrate the importance of de-gassing in the enhancement of dispersion of fine oil droplets in water. Specifically, these results support the view that de-gassing, not the freeze-thaw process itself, enhances these emulsions. These results are also supported by earlier results on the enhanced dispersion of hydrophobic solids in de-gassed water. These results and those on the effect of de-gassed water on gassed oils and on the re-dispersion of sealed, phase-separated liquids, form a body of evidence in support of the important role of de-gassing. Given that freezing is known to cause de-gassing, leads to the interpretation of freeze-thaw enhanced dispersion as an effect due to local de-gassing via freezing. There is no doubt that the freeze/thaw process also offers an efficient means of mixing oil and water; however, it seems clear that since de-gassing by itself can create the enhanced dispersion observed, the simplest explanation is that freezing creates local de-gassing and hence enhances dispersion.

\section{2. 'Spontaneous' dispersions}

The original study [1] indicated that some oils can have a higher turbidity when formed 'spontaneously' compared with shaking or with shaking after phase separation, or vice versa. Given the limited range of data on different systems it is difficult at this stage to understand the detailed mechanisms involved. Separately de-gassed squalane and water produced dispersions, on shaking, very similar to those observed on shaking de-gassed mixtures (see Fig. 5). However, PFH separately de-gassed, pro- duced significantly lower turbidity mixtures. The fluorocarbon results are further complicated by the similarity of the refractive indices and the nature of the turbidity measurements. Although PFH and water have very close refractive indices, the turbidimeter, which measures scattered visible light, still registers a significant difference. The separately de-gassed PFH and water mixtures were visibly cloudy upon shaking (see Fig. 2). This visible cloudiness was probably due to light absorption, rather than scattering, which would explain the relatively low NTU values observed (see Fig. 3). The droplet sizes, of about $0.5 \mu \mathrm{m}$, are in the range where interpretation of the turbidity data is difficult.

'Spontaneous' dispersions are produced by four or five cycles of a freeze/pump/thawing process, which will effectively expose the mixtures to fairly vigorous mixing conditions, both mechanical and thermal, over a long period of time, say up to $45 \mathrm{~min}$. By comparison, separately de-gassed mixtures are only manually shaken together for, say, $10 \mathrm{~s}$. The two processes are obviously very different and it is perhaps surprising that some oils give comparable turbidity curves for these two extreme cases.

The effects of the different types of mixing will most likely depend on the physical properties of the oil. This is because of the nature of the freeze-thaw de-gassing process. During rapid freezing under liquid nitrogen, ice formation causes dissolved molecular gases to nucleate into fine bubbles, which can be seen to be trapped in the ice. On melting, these bubbles are released in a continuous upwards flow from the ice/water boundary. Hence, the fact that the fluorocarbon oil (PFH) is much denser than water, while the hydrocarbon squalane is less dense (see Table 2), makes a significant difference to the de-gassing process. This density difference can lead to a mechanism whereby squalane, being above the water phase, could re-gas as the dissolved gas is liberated from the (lower) melting water phase. This would mean that squalane would not be able to de-gas as much in the early stages of de-gassing. This could lead to a lower 'spontaneous' dispersion. By comparison, $\mathrm{PFH}$ cannot re-absorb the gas from the water, because it is at the bottom of the tube. This would mean that the initial cycles of de-gassing for PFH would be more effective than for squalane and thus a greater 'spontaneous' dispersion could be formed.

However, earlier studies with dodecane [1], another hydrocarbon, show results comparable to those of PFH not squalane. This similarity between PFH and dodecane, may be related to their similar melting points, and their being relatively close to water (see Table 2). This means that when water is melting and releasing trapped dissolved gases, the dodecane is still solid (at the top of the tube) and thus the released gases cannot dissolve into the upper (solid) oil phase. Squalane, on the other hand, has a very low melting point, hence the released dissolved gases will be captured in the melted, liquid squalane at the top of the

Table 2

Densities and melting points for several oils used in these studies

\begin{tabular}{llc}
\hline & Density $(\mathrm{g} / \mathrm{ml})$ & Melting point $\left({ }^{\circ} \mathrm{C}\right)$ \\
\hline Perfluorohexane $(\mathrm{PFH})$ & 1.669 & -4 \\
Squalane & 0.809 & -38 \\
Dodecane & 0.755 & -9.6 \\
\hline
\end{tabular}


tube and its 'spontaneous' dispersion, during the freeze-thaw process, will be suppressed.

\subsection{Theoretical stability of PFH dispersions}

The experimental data obtained for de-gassed dispersions of perfluorohexane (PFH) combined with the zeta potential for the similar fluorocarbon liquid PFOB [4], can be used to illustrate the expected colloidal stability of these dispersions. Although the dispersion is meta-stable, colliding droplets are kept apart by the electrostatic double layer repulsion between two colloidal particles. Combination of this electrostatic repulsion with the van der Waals attraction between the droplets is called the DLVO theory. An approximate equation [10] for this interaction energy, $V_{\mathrm{s}}$, is given by:

$\frac{V_{\mathrm{S}}}{k T}=\frac{a}{k T}\left[2 \pi \varepsilon_{0} D \psi_{0}^{2} \exp (-\kappa H)-\frac{A_{121}}{12 H}\right]$

where $a$ is the average droplet radius, $\varepsilon_{0}$ the permittivity of free space, $D$ the static dielectric constant, $\psi_{0}$ the surface or zeta potential on the droplet (measured to be $50 \mathrm{mV}$ ), $H$ the separation distance between two interaction droplets, $\kappa^{-1}$ the Debye length of the electrolyte solution and $A_{121}$, the Hamaker constant (which for fluorocarbon/water/fluorocarbon is about $0.3 \times 10^{-20} \mathrm{~J}$ ). The interaction energy calculated using the parameters obtained for PFH droplets in water is given in Fig. 6. Clearly, collisions between droplets with kinetic energies even up to $10 \mathrm{kT}$ or more will have no effect on the stability of the droplets and no coalescence is expected, as observed here and in earlier reports [1-4]. This repulsive energy is the origin of the dispersion stability after its formation. However, gravitational forces will slowly cause phase separation or settling, as discussed in the following section.

\subsection{Droplet coalescence with the oil phase}

Observations on perfluorohexane and on several other oils have shown that over a period of days the oil droplets settle (or rise) under the action of gravity. However, the fine droplets may not completely re-coalesce with the oil phase. Re-coalescence is often observed in other systems [11] but is interesting here because it does not occur between randomly colliding droplets

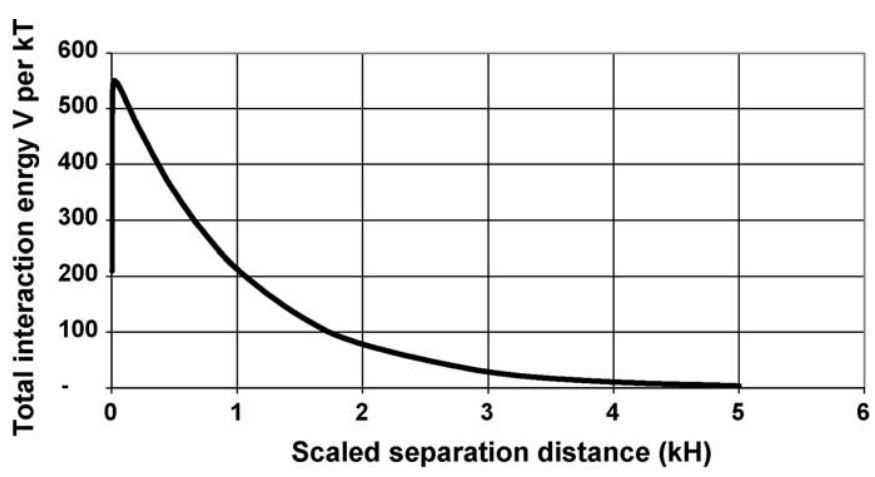

Fig. 6. DLVO interaction curve for two fluorocarbon droplets (such as PFH) in water. freely moving in the aqueous phase [2] and hence, may not occur, even slowly, at the droplet-bulk phase boundary. In some of the early experiments, a fine cloudy layer was seen underneath the excess oil (when less dense than water) at the top of the mixture, following freeze-thaw de-gassing and after settling for some time in a sealed tube (the settling time depends on the density of the oil). This was most likely due to ambient vibrations at the liquid phase boundary continually dispersing fine oil droplets or fine droplets not re-coalescing with the oil phase once formed. However, when the glass tubes were thoroughly re-cleaned this cloudiness often disappeared. Thus, the effect may be related to the hydrophobicity of the walls of the tube and the drainage between oil droplets and an oil phase. It is interesting that these effects are not observed on shaking normal (gassed) mixtures because no fine droplets are created.

We can compare the electrostatic double layer barrier force between a fluorocarbon oil droplet and a flat surface with the gravitational force. The expected double layer barrier will be typically about $1 \mathrm{mNm}^{-1}$ [10], which gives an electrostatic barrier force for a droplet of $0.3 \mu \mathrm{m}$ radius of $3 \times 10^{-10} \mathrm{~N}$. By comparison, the gravitational force acting on the droplet is given by $(4 / 3) \pi R^{3} \Delta \rho g$, which for a density difference of $670 \mathrm{~kg} \mathrm{~m}^{-3}$, gives a force of about $8 \times 10^{-16} \mathrm{~N}$. This simple analysis suggests that charged oil droplets will not readily coalesce with the oil phase simply by settling, even with many layers of oil droplets adding their buoyancy forces. The van der Waals attraction for this system is weak and only acts over a very short range. The hydrophobic attraction might reduce the magnitude of the double layer but it has been shown previously [2] that this force is not strong enough to affect a significant double layer repulsion with a long Debye length (i.e. as expected in distilled water) and, in any case, it is likely that no such attraction is present for these de-gassed mixtures.

The electrical double layer barrier is strongly dependent on the electrostatic potential $\left(\psi_{0}\right)$ at the droplet surface. This potential depends directly on the particle charge. Is it possible that the coalescence sometimes observed is due to the continuous gravity force on the droplet acting to force the mobile surface charges apart locally? The electrostatic potential on typical oil droplets is about $-50 \mathrm{mV}[4,8]$ and this corresponds to a charge density of $-0.0008 \mathrm{Cm}^{-2}$ for a Debye length of $50 \mathrm{~nm}$. This charge density equates to a surface with charges spaced about $16 \mathrm{~nm}$ apart, at equilibrium. Can this effective density be reduced, given the curvature of the oil droplets to allow coalescence via van der Waals forces. This situation, drawn roughly to scale, is illustrated in Fig. 7. Coalescence is, of course, thermodynamically favored and these charges, which should be surface mobile,

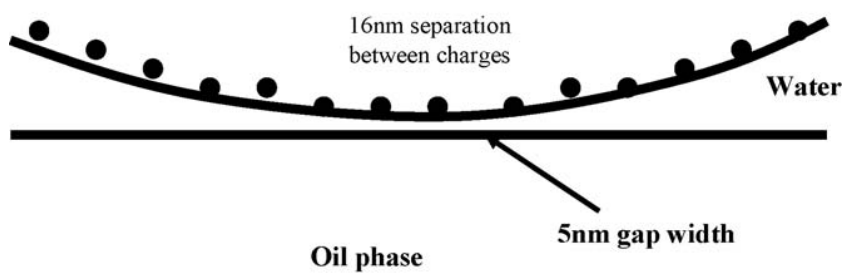

Fig. 7. Representation of oil droplet coalescing with oil phase. 
could be pushed apart to allow the surfaces to contact via van der Waals forces. It is clear that a slightly higher compression of the charges over the rest of the droplet would be required to free up the areas within the contact region (see schematic diagram in Fig. 7). If this mechanism is accepted, then the lack of coalescence for droplets colliding in solution must simply be due to the much smaller time scale involved in collisions. That is, the surface charges (adsorbed hydroxyl ions) cannot move away fast enough because in doing so they must compress the other ions on the droplet surface. This will create a repulsive barrier during (rapid) droplet collisions, which may be reduced when droplets are in long-term contact with the bulk oil/water interface.

\subsection{Droplet dispersion by mechanical and thermal agitation}

It is interesting to estimate the total volume percent of fine oil droplets which could be dispersed in water at $\mathrm{pH} \mathrm{7,} \mathrm{that} \mathrm{is}$ for either nitrogen gassed or de-gassed water, given the requirement for adsorption of hydroxyl ions. The observed 'natural' charge on oil droplets in water is about $-0.0008 \mathrm{Cm}^{-2}$. If we assume that the water acts as a reservoir which limits hydroxyl adsorption once the $\mathrm{pH}$ has decreased by 1 unit, then we have a potential reservoir for adsorption of $10^{-6} \mathrm{M}$ hydroxyl ions. Assuming that the oil droplets have a radius of $0.3 \mu \mathrm{m}$, this corresponds to a possible volume fraction of about $1 \%$ oil in water, which is generally close to the level observed. This analysis is also supported by the increased turbidity observed for PFH dispersions produced at higher $\mathrm{pH}$ values.

The limited data available $[2,4]$ suggests that the oil droplets produced by the de-gassing process are fairly uniform in size at around $0.3-0.4 \mu \mathrm{m}$ radius. However, if the oil has a slight solubility in water, Ostwald ripening can create larger droplets and a wider size distribution [2]. It is remarkable that the simple process of thermal and mechanical shaking or just mechanical agitation can produce a fine particle size distribution (compared with samples not de-gassed) for water insoluble, hydrophobic oils, such as perfluorooctyl bromide (see Fig. 8). Mechanical or thermal agitation can introduce mixing energies beyond the

\section{Charges per oil droplet}

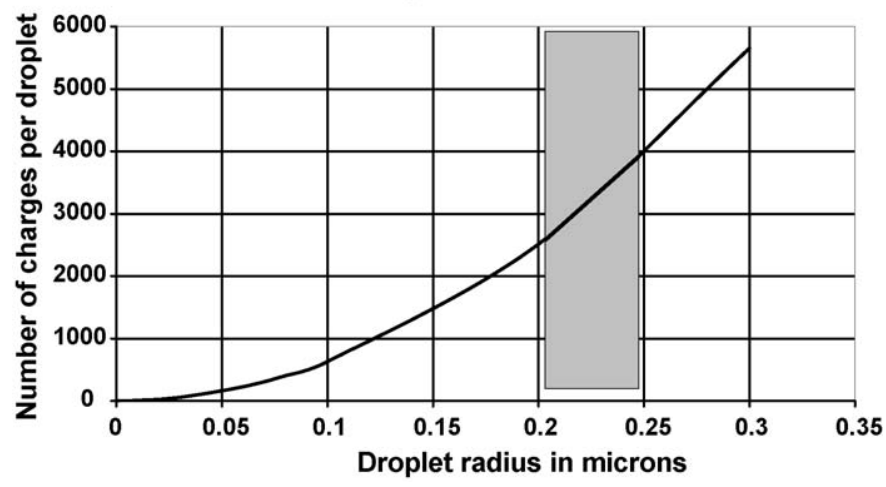

Fig. 8. Graph showing number of charges per fluorocarbon droplet (the important size range is between 0.2 and $0.25 \mu \mathrm{m}$, from particle size distribution data). thermal kinetic energy level, kT. In the following section we consider the work required to break droplets away from an oil-water interface.

The energy required to create a spherical oil droplet of radius $R$ from an oil phase can be calculated from the surface work done in creating the droplet. This is simply given by the relation: $4 \pi R^{2} \gamma_{\text {int }}$, where $\gamma_{\text {int }}$ is the oil-water interfacial energy. This relationship favours the release of small droplets. Assuming a droplet has been almost completely formed, the breakaway energy could be roughly estimated from the theory of adhesion of deformable spherical objects to flat surfaces [12]. In which case, the pull off force, $P$, is given by $P=3 \pi R \gamma_{\text {int }}$. If we make the assumption that the pull off force has to extend say, $3 \mathrm{~nm}$ for the droplet to be released, the energy involved can be easily estimated as $P \times 3 \times 10^{-9} \mathrm{~J}$. As an example, the work done to release a $5 \mathrm{~nm}$ radius oil droplet $\left(\gamma_{\text {int }} 50 \mathrm{~mJ} \mathrm{~m}^{-2}\right)$ will be about $2000 \mathrm{kT}$, which is not unreasonable for the mechanical energies involved in vigorous shaking. Droplets of this size, also have about this level of surface energy. However, larger droplets require huge amounts of work. For example, the pull off energy for a micron diameter oil droplet is of the order of $10^{5} \mathrm{kT}$ and the surface work about $4 \times 10^{7} \mathrm{kT}$. These rough values indicate that mechanical/thermal agitation will favor the release of large numbers of small oil droplets.

These small droplets will be unstable to collisions with other droplets because the (equilibrium hydroxyl) charge density is relatively low and so surface coverage of the spherical particle will be sparse and most collisions will cause coalescence. However, as the particle grows the charge coverage of the sphere will reach a point where even collisions with small, primary droplets will not allow coalescence and the particles will grow no further. The number of charges per oil droplet is given as a function of droplet size in Fig. 8. It is likely that this sort of limited growth model will produce a relatively narrow particle distribution, as observed for vigorously shaken PFOB dispersions (having a narrow particle size distribution around $0.3 \mu \mathrm{m}$ in radius). The experimentally observed size distributions suggest that the charge coverage needed to prevent further growth is of the order of a few thousand charges per droplet, which appears reasonable. Of course, it should be realized that very large oil droplets produced during shaking rapidly leave the system under the action of gravitational forces.

\subsection{Conditions for cavitation}

It has been proposed that the fundamental cause of enhanced dispersion on de-gassing is due to the cavitation of hydrophobic surfaces when pulled apart in water. Following this assumption, we can develop thermodynamic criteria to predict what systems will be affected by de-gassing. In this, it is perhaps useful to think in terms of a theoretical or ideal water contact angle, which can be calculated for a water droplet placed on any (assumed flat and rigid) liquid surface. Thus, for a water droplet on hexadecane the theoretical contact angle can be obtained using the data in Table 1 and the Young equation:

$\gamma_{\mathrm{sv}}=\gamma_{\mathrm{lv}} \cos \theta+\gamma_{\mathrm{sl}}$ 
Table 3

Surface tension criteria for cavitation

\begin{tabular}{lll}
\hline Dispersion system & $\begin{array}{l}\text { Criterion: } \gamma_{\mathrm{dp} / \mathrm{v}}+ \\
\gamma_{\mathrm{dm} / \mathrm{v}}>\gamma_{\mathrm{int}}>\gamma_{\mathrm{dp} / \mathrm{v}}\end{array}$ & $\begin{array}{l}\text { Cavitate on } \\
\text { separation? }\end{array}$ \\
\hline Fluorocarbon dispersed in water & $15+73>42>15$ & Yes \\
Hydrocarbon dispersed in water & $24+73>53>24$ & Yes \\
Water dispersed in hydrocarbon & $73+24>53<73$ & No \\
Hydrocarbon dispersed in silicone & $24+19>10<24$ & No \\
Silicone dispersed in hydrocarbon & $19+24>10<19$ & No \\
\hline
\end{tabular}

where, in this case the theoretical water contact angle is $111^{0}$. Fluorocarbon liquids also give high theoretical water contact angles that are significantly above $90^{\circ}$. It is therefore expected that these liquids should cause cavitation as they are separated in water, because the gas/vapour interface with the oil actually has a lower surface energy than the oil/water interface. That is, these oils would rather form an interface with gas or vapor, than with liquid water.

Thus, in general, the condition for cavitation is given by:

$\gamma_{\mathrm{dp} / \mathrm{v}}+\gamma_{\mathrm{dm} / \mathrm{v}}>\gamma_{\mathrm{int}}>\gamma_{\mathrm{dp} / \mathrm{v}}$

where $\gamma_{\mathrm{dp} / \mathrm{v}}$, is the interfacial tension between the dispersed phase and vapour; $\gamma_{\mathrm{dm} / \mathrm{v}}$ between the dispersion medium and vapour and $\gamma_{\text {int }}$ between the dispersed phase and dispersion medium. Some examples are given in Table 3. The application of this criterion can be used to predict which dispersions will produce cavitation and hence be affected by de-gassing. The results predict, for example, that the dispersion of silicone oil in hydrocarbon oil (or vice versa) will not be affected by degassing. Also, water droplets dispersed in hydrocarbon oil will be unaffected by de-gassing (Table 3).

\subsection{Applications}

The results reported here and in several earlier papers indicate that the enhancement of oil dispersion in water on de-gassing is a general phenomenon which has some obvious potential applications in industry, such as in pharmaceuticals, where there is the possibility of reducing the side effects of dispersants, such as surfactants [4]. Also, the effect raises the possibility of detergent-free cleaning [3], which might be of importance in the development of nano-technology. Hence, it is important to understand the precise conditions under which the phenomenon occurs.

Although the enhanced dispersion is clearly established, the degree of de-gassing required to reduce cavitation has yet to be studied in detail. Earlier results [13] indicated that the level of de-gassing produced by a typical water-jet pump (about
$97 \%$ de-gassing) is not sufficient to produce significant levels of enhanced dispersion, whereas de-gassing at the $99.999 \%$ is effective. The limited results obtained here on the sequential freeze-thaw cycles of PFH and water indicates that a range of effects are possible, depending on the de-gassing level. However, it is not yet known whether significant enhancement of dispersion occurs sharply or over a gradual transition with the level of de-gassing. Lastly, it is also not known if further de-gassing, say to ultra-high vacuum levels, will enhance dispersion still further.

\section{Conclusions}

The results presented in this work support the hypothesis that de-gassing causes the enhanced dispersion of oils and hydrophobic powders in water. Once dispersed, the particles appear to become charged and do not coalesce. They can generally be re-dispersed, following gravity settling, if sealed under vacuum. This enhanced dispersion seems to be applicable to any hydrophobic oil or solid and offers potential for commercial applications in several areas.

\section{Acknowledgement}

The authors would like to thank Mr Jeffrey Lai for measuring interfacial tensions.

\section{References}

[1] R.M. Pashley, J. Phys. Chem. B 107 (7) (2003) 1714-1720.

[2] N. Maeda, K.J. Rosenberg, J.N. Israelachvili, R.M. Pashley, Langmuir 20 (8) (2004) 3129-3137.

[3] R.M. Pashley, M. Rzechowicz, L.R. Pashley, M.J. Francis, J. Phys. Chem. B 109 (2005) 1231-1238.

[4] M.J. Francis, R.M. Pashley, Colloids Surf. A: Physiochem. Eng. Aspects 260 (2005) 7-16.

[5] V.V. Yaminsky, V.S. Yushchenko, E.A. Amelina, E.D. Shchukin, J. Colloid Interface Sci. 96 (2) (1983) 301-306.

[6] R.M. Pashley, P.M. McGuiggan, B.W. Ninham, D.F. Evans, Science 229 (1985) 1088-1089.

[7] H.K. Christenson, P.M. Claesson, Science 239 (1988) 390-392.

[8] K.G. Marinova, R.G. Alargova, N.D. Denkov, O.D. Velev, D.N. Petsev, I.B. Ivanov, R.P. Borwankar, Langmuir 12 (8) (1996) 2045-2051.

[9] G.R. Burnett, R. Atkin, S. Hicks, J. Eastoe, Langmuir 20 (2004) 5673-5678.

[10] R.M. Pashley, M.E. Karaman, Applied Colloid and Surface Chemistry, J. Wiley, New York, 2004.

[11] E. Dickinson, J. Chem. Soc. Faraday Trans. 84 (1988) 871.

[12] K.L. Johnson, K. Kendall, A.D. Roberts, Proc. R. Soc. A 324 (1971) 301.

[13] M.E. Karaman, B.W. Ninham, R.M. Pashley, J. Phys. Chem. 100 (1996) 15503-15507;

See also:. M. Alfridsson, B. Ninham, S. Wall, Langmuir 16 (26) (2000) 10087-10091. 